\title{
Simulation of a Laboratory Scale Ball Mill via Discrete Element Method Modelling
}

\author{
Mohsen Mhadhbi \\ Laboratory of Useful Materials, National Institute of Research and Physicochemical Analysis, Technopole Sidi Thabet, \\ Ariana, Tunisia \\ Email: mohsen.mhadhbi@inrap.rnrnt.tn
}

How to cite this paper: Mhadhbi, M (2021) Simulation of a Laboratory Scale Ball Mill via Discrete Element Method Modelling. Advances in Materials Physics and Chemistry, 11, 167-175.

https://doi.org/10.4236/ampc.2021.1110016

Received: August 25, 2021

Accepted: October 22, 2021

Published: October 25, 2021

Copyright (C) 2021 by author(s) and Scientific Research Publishing Inc.

This work is licensed under the Creative Commons Attribution-NonCommercial International License (CC BY-NC 4.0). http://creativecommons.org/licenses/by-nc/4.0/

\begin{abstract}
Discrete Element Method (DEM) is a powerful tool for simulating different types of mills. It also used for computing different types of particles such as rocks, grains, and molecules. DEM has been widely used in the field of rock mechanics. In the present work, DEM approach is applied to model the milling media (powder particles and balls) inside a planetary ball mill and to estimate the distribution of particles of a dry powder during milling. In fact, the efficiency of the DEM strongly depends on the input parameters. The DEM simulation results indicated that DEM is a promising tool for the simulation of the dynamic particles motion and interactions within planetary ball mill. These results could be utilized to further develop the synthesis performance, anticipate the reaction, and reduce the wear in the dry milling reactions.
\end{abstract}

\section{Keywords}

Modelling, Discrete Element Method, Milling Media, Planetary Ball Mill, Simulation

\section{Introduction}

Simulation of various types of mills through DEM method provides the occasion to understand the dynamics inside the mill and also to develop the mill design and operation [1]. In 1979, Cundall and Strack [2] were the first who proposed the DEM to simulate the rock fracture problem. Mishra and Rajamani [3] [4] were introduced DEM method to simulate the motion of balls charge in the tumbling mills. After that, Cleary [5] studied the charge motion, segregation, wear, and power draw in a ball mills through DEM simulation.

Several studies have been reported on modelling of mills by DEM simulations. For example, Xie et al. [6] studied the effect of filling level on the vibration and 
wear of a semi-autogenous grinding (SAG) based on the collision energy on liner. They concluded that the high energy collision between grinding media and liner was the principal cause for mill vibration and liner wear. DEM simulations were applied to study how shell liner can induce ball segregation in a ball mill with four sections and three ball size classes [7]. It has been showed that the change of axial liner profile configuration can influence ball segregation, notably at $75 \%$ of critical speed. Orozco et al. [8] investigated the evolution of granular materials in a rotating drum partially filled with powders and balls by using DEM simulations. They revealed that when the energy dissipation increases, due to the inelastic collisions between the balls, the grinding process becomes less energetic for a larger amount of balls. Jiang et al. [9] studied DEM simulations of particle motion in different ball mills based on similarity. The result showed that the detaching angles stay identical by modifying the gravitational acceleration and the rotational speed. The DEM modelling of liner evolution and its effect on grinding rate in ball mills using EDEM software was also reported [10]. A breakage rate model was also developed for correlating liner profile to mill performance. The obtained result revealed that the combination of the prediction of liner profile and grinding rate exhibited promise a significant tool for mill liner design. Stoimenov and Ruzic [11] used EDEM software for simulating the attritor mill to investigate motion and trajectory of balls and particles during milling. It was concluded that the amount of contacts between balls and particles strongly depends on particle radius.

Furthermore, it has been found that the simulation of the attritor mill could contribute to decreasing milling time and to detail the motion of the balls and powders inner the mill [12]. Cleary et al. [13] particularly revealed that the use of superquadratic shape was capable to improve the predictions for charge structure in an SAG mill, which are near to those measured experimentally. Additionally, Cleary and Hoyer [14] showed good agreement between DEM simulation and experiment for the case of a centrifugal mill. However, a comparative study between positron emission particle tracking (PEPT) derived charge features in wet tumbling mill and friction adjusted DEM model was conducted by Govender et al. [15]. They concluded that the flow was not specifically sensitive to the exact choice. Slight differences between PEPT and DEM were also noticed. Similarly, a multiscale method for involving fine particle effects in DEM models of grinding mills was proposed [16]. It has been found that the presence of the powder can strongly influence the damping behaviour of the grinding media especially at smaller confining bed pressure and at higher shear rate. In a previous work [17], we investigated the simulation of ball milling process through DEM modelling. The simulation results showed the efficiency of DEM to improve the performance and efficiency of the mill.

In this paper, DEM approach was applied in a very sample way to investigate the planetary ball milling process including trajectory, velocity and distribution of particles powder and balls inside the mill. 


\section{DEM and Fundamental Principle}

\subsection{Contact Dynamics Model}

DEM is a particle based technique where each particle in the flow is tracked and all collisions between particles and between particles and boundaries are modeled using a contact force low.

The contact force law is based on a linear spring-dashpot model defined by normal $\left(F_{n}\right)$ and tangential $\left(F_{t}\right)$ components.

The normal force is given by the following Equation:

$$
F_{n}=-k_{n} \Delta x+C_{n} \vartheta_{n}
$$

where $k_{n}$ is the normal spring stiffness constant $\left(k_{n}=\frac{4}{3} E^{*} \sqrt{R^{*} \delta_{N}}\right), \Delta x$ is the amount of overlap, $\mathcal{\vartheta}_{n}$ is the normal velocity, and $C_{n}$ is the normal damping coefficient $\left(C_{n}=2 \sqrt{\frac{5}{6}} \beta \sqrt{S_{n} m^{*}}\right)$, which determines the effective coefficient of restitution.

In addition, the tangential force is given by:

$$
F_{t}=\min \left(\mu F_{n}, k_{t} \int \vartheta_{t} \mathrm{~d} t+C_{t} \vartheta_{t}\right)
$$

where $k_{t}$ is the tangential spring stiffness constant $\left(k_{t}=8 G^{*} \sqrt{R^{*} \delta_{n}}\right), C_{t}$ is the tangential damping coefficient $\left(C_{t}=2 \sqrt{\frac{5}{6}} \beta \sqrt{k_{t} m^{*}}\right), \mu$ is the friction coefficient, and $\vartheta_{t}$ is the tangential velocity. The integral of the tangential velocity over the relative behaves as an incremental spring that stores energy from the relative tangential motions. The total tangential force is limited by the Coulomb's frictional limit $\left(\mu F_{n}\right)$.

\subsection{Particle Shape}

During DEM simulation, the particles are represented as a spheres or super-quadrics according to the following equation:

$$
\left(\frac{x}{a}\right)^{m}+\left(\frac{y}{b}\right)^{m}+\left(\frac{z}{c}\right)^{m}=1
$$

where $m$ is a constant that determines the shape of the particles and the ratios $b / a$ and $c / a$ determine the aspect ratios of the particles.

- For $m=2$ : elliptical particles are obtained.

- For $m=10$ and aspect ratios $=1$ : cubic particles are obtained.

- For $a=b=c$. spherical particles are obtained (our case during this study).

The simulation time using super-quadratics is much longer than those using spheres.

Williams and Pentland [18] first used DEM simulations for modeled 2D shape particles. However, Cleary [19] first applied DEM modelling to investigate 3D shape particles. 


\subsection{Flow Chart of DEM Algorithm}

The DEM algorithm used in simulations is composed of nine steps. The first step is to input a DEM data file. The second step is to use important geometry. The third step is to sets up particle group. The fourth step is to calculate particle to particle and particle to boundary interactions. The fifth step is to search neighboring particles and boundaries. The sixth step is to calculate acceleration and velocity. The boundary condition is also determined in the seventh step. The eighth step is to calculate density. The ninth step is to modify particle position, velocity, and time step. It all of the steps are carried out and if the time (T) is greater than the final time (Tend), the program exits the loop and saves the processed DEM. Otherwise, the program goes back to the first step.

\section{Mill Configuration and Simulation Parameters}

In this study, we used a planetary ball millin laboratory scale. The powders distribution, the balls distribution, and the wear were modeled using DEM. The simulations of dry mill were conducted by using a standard coefficient of restitution of 0.3 and a friction coefficient of 0.75 (ball-ball and ball-liner collisions) [20]. The charge consisted of powders and balls with filling of $40 \%$ of the charge (by volume). The specific gravity of the media is equal to 2.7 . However, the materials properties used for simulation are presented in Table 1.

The ball mill parameters used for simulation are illustrated in Table 2.

Table 1. Materials properties used for simulation.

\begin{tabular}{cc}
\hline Parameter & Value \\
\hline Poiasson's ratio & 0.3 \\
Young modulus $\left(\mathrm{N} / \mathrm{m}^{2}\right)$ & $1.8 \times 10^{11}$ \\
Density $\left(\mathrm{kg} / \mathrm{m}^{3}\right)$ & 7700 \\
\hline
\end{tabular}

Table 2. Ball mill parameters.

\begin{tabular}{cc}
\hline Parameter & Value \\
\hline Shaft power $(\mathrm{kW})$ & 0.37 \\
Angular speed $(\mathrm{rpm})$ & 250 \\
Effective diameter of disc $(\mathrm{mm})$ & 140 \\
Mill filling (\%) & 40 \\
Mill speed (\% critical speed) & $10-100$ \\
Time step (s) & $1.01 \times 10^{-4}$ \\
Ball density $\left(\mathrm{kg} / \mathrm{m}^{3}\right)$ & 7700 \\
Vial density $\left(\mathrm{kg} / \mathrm{m}^{3}\right)$ & 7700 \\
Ball size $(\mathrm{mm})$ & 15 \\
Length $(\mathrm{mm})$ & 370 \\
Depth $(\mathrm{mm})$ & 530 \\
Height $(\mathrm{mm})$ & 500 \\
Weight $(\mathrm{kg})$ & 35 \\
\hline
\end{tabular}




\section{DEM Simulation Results}

The modelling hypotheses are described by starting with the identification of the material (contact law), the geometry of mill and filling, and the description of the simulation and the post-processing. During simulation, all the particles are considered and represented as a spherical element.

The stages of building a model are:

- Geometry clean-up;

- Geometry import;

- Settings the dynamics of model elements;

- Setting the parameters of model elements;

- Setting the parameters of bulk materials.

Moreover, the computer aided design (CAD) geometry used for DEM simulations is presented in Figure 1. The geometry shows the characteristics regions of the charge motion and the stochastic variability of the particles flow pattern. Thus, the particles are colored based on their velocity.

Figure 2 illustrates the different stages of particle breakage. The particle size distribution is mainly concentrated near the wall of vial due to the high centrifugal accelerations caused by the movement of the vial. With an increase in velocity, the powders occupy almost the entire volume of the vial space. In addition, the smaller particles, which receive large amount of the impact energy, circulate on closed trajectories near the wall of vial due to gravity. Thus, the simulation results are in accordance with those obtained by Hirosawa et al. [21].

Moreover, the speed coloring (from blue: slow to red: fast) provides information on the charge motion (Figures 2(a)-(d)). At the beginning (Figure 2(a)), it appears that all particles are distributed uniformly inside the charge. As the mill speed increases (Figure 2(b) and Figure 2(c)), particles concentrate beside the wells and launched higher from the shoulder of the charge. However, it does mean that the particles and balls are well mixed. Figure 2(d) presents moving

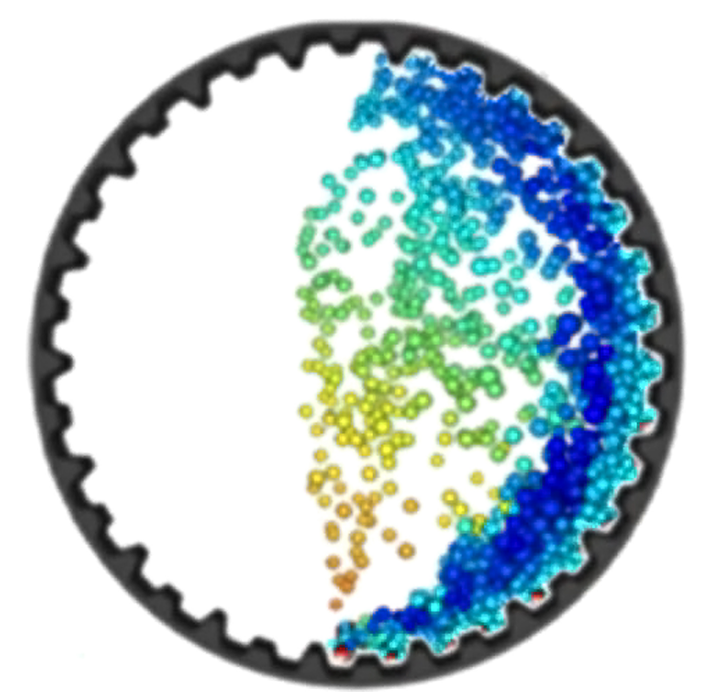

Figure 1. CAD geometry used for DEM simulation. 


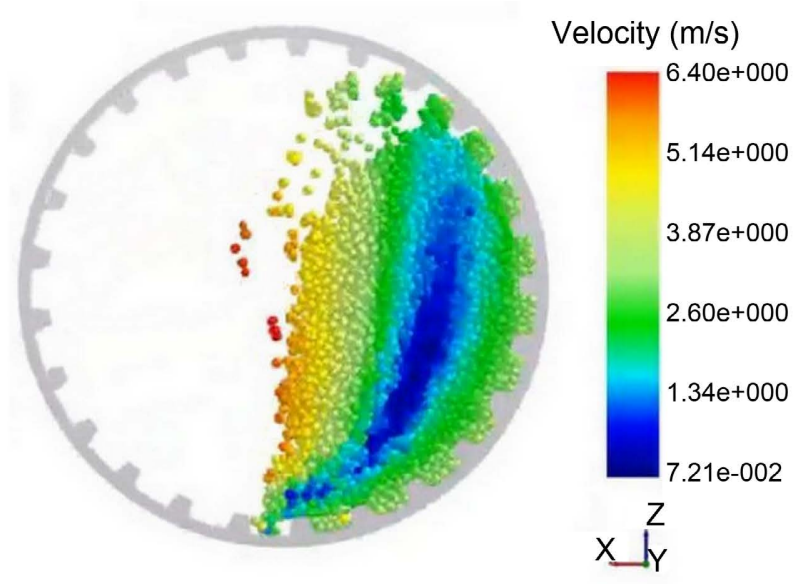

(a)

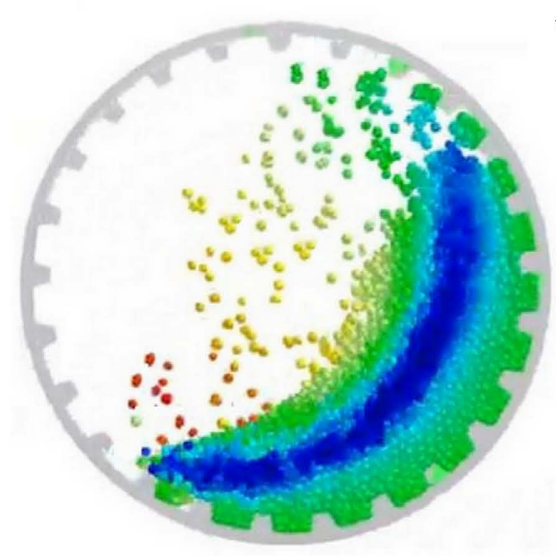

(c)

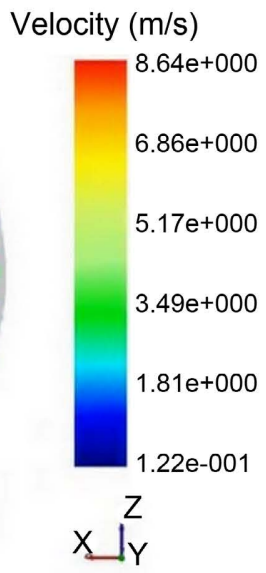

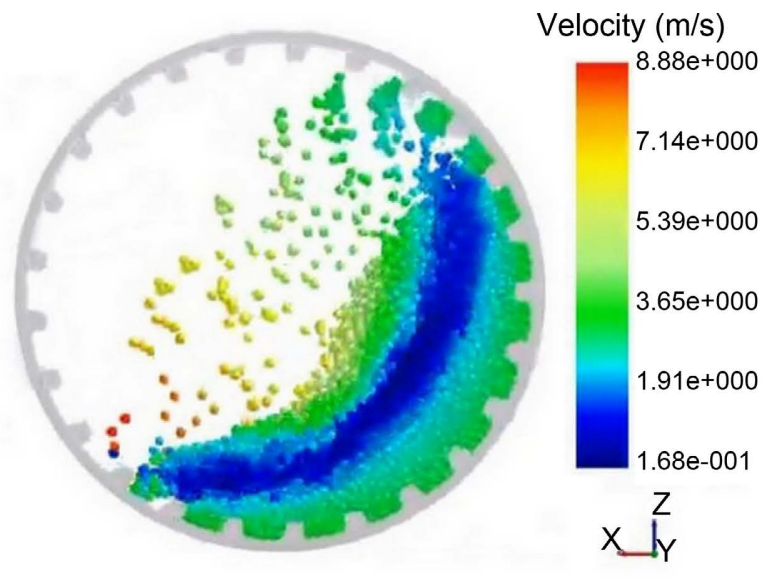

(b)

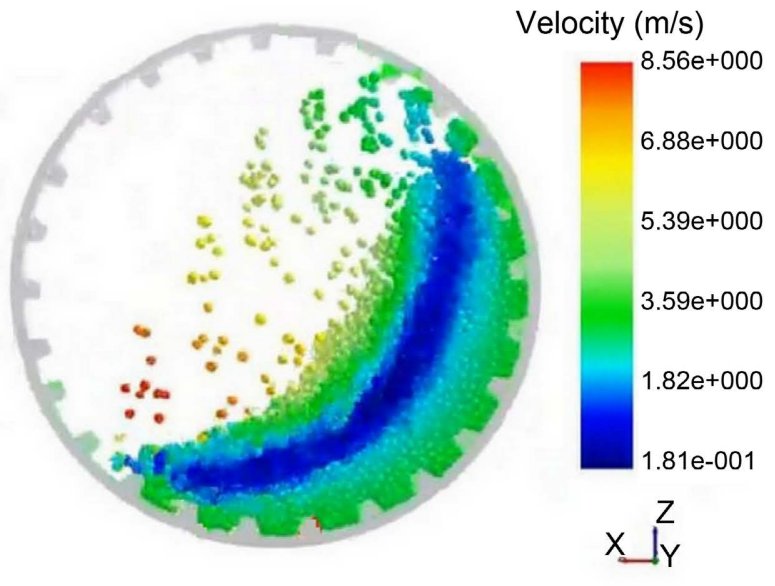

(d)

Figure 2. Snapshots of particles in planetary mill at different stages of particle breakage.

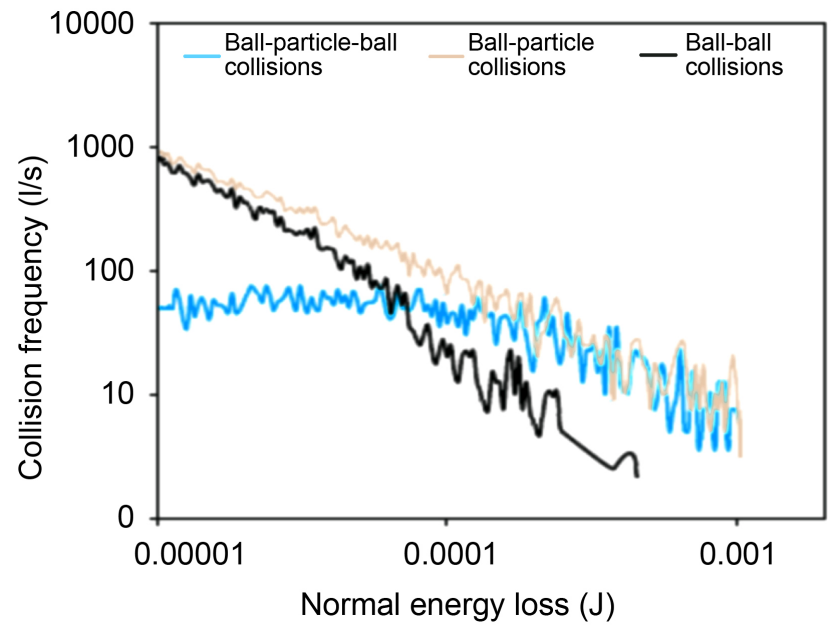

Figure 3. Variation of collision frequency with energy loss for different types of collisions.

particles in high speed, which produce high energy impacts during milling process (with a velocity of $8.56 \mathrm{~m} / \mathrm{s}$ ). That may be explained by the fact that the 
trickling of the finer powder particles through the milling media (walls and balls). It would be also considered that the number of collisions decreases with increasing energy per collision [22].

The variation of collision frequency with energy loss for different types of collisions (ball-particle-ball, ball-ball, and ball-particle), collected from DEM simulation, is shown in Figure 3. The figure demonstrates a reduction in the number of collisions and an increase in their magnitude. In addition, for higher values of energy loss the higher magnitude binaries were converted into ternary collisions.

\section{Conclusion}

The investigation of ball mill behavior through DEM has been extended to include the coarse end of the powder particles. DEM simulation provides a powerful tool for understanding the effect of the variables on planetary ball mill performance. The combination of an increase in energy intensity and collision frequency leads to increased probability of breaking powder particles. Indeed, high energy and low frequency impacts can cause body breakage. By optimizing the calibration and design through starting materials, a number of enhancements can be made to reduce relining time and to improve wear resistance. DEM approach can be applied to calculate collision rates and impact energy spectra of ball mills in industrial scale. It has also gained common acceptance for understanding the behavior of particles inside the mill. Future study on the milling conditions can enhance the mechanical milling reactions in order to appropriately produce unstable materials at room temperature.

\section{Acknowledgements}

The author would like to thank Ms. Yavonne Zhang and Ms. Melissa Luo, AMPC Editorial Assistants, for their assistance.

\section{Conflicts of Interest}

The author declares no conflicts of interest regarding the publication of this paper.

\section{References}

[1] Cleary, P.W. (2001) Recent Advances in DEM Modelling of Tumbling Mills. Minerals Engineering, 14, 1295-1319. https://doi.org/10.1016/S0892-6875(01)00145-5

[2] Cundall, P.A. and Strack, O.D.L. (1979) A Discrete Numerical Model for Granular Assemblies. Geotechnique, 29, 47-65. https://doi.org/10.1680/geot.1979.29.1.47

[3] Mishra, B.K. and Rajamani, R.K. (1992) The Discrete Element Method for the Simulation of Ball Mills. Applied Mathematical Modelling, 16, 598-604. https://doi.org/10.1016/0307-904X(92)90035-2

[4] Mishra, B.K. and Rajamani, R.K. (1994) Simulation of Charge Motion in Ball Mills. Part 1: Experimental Verifications. International Journal of Mineral Processing, 40, 171-186. https://doi.org/10.1016/0301-7516(94)90042-6

[5] Cleary, P.W. (1998) Predicting Charge Motion, Power Draw, Segregation and 
Wear in Ball Mills Using Discrete Element Methods. Minerals Engineering, 11, 1061-1080. https://doi.org/10.1016/S0892-6875(98)00093-4

[6] Xie, C., Zhao, Y., Song, T. and Zhao, Y. (2021) Investigation of the Effect of Filling Level on the Wear and Vibration of a SAG Mill by DEM. Particuology.

https://doi.org/10.1016/j.partic.2021.04.009

[7] Chimwani, N. and Bwalya, M.M. (2020) Using DEM to Investigate How Shell Liner can Induce Ball Segregation in a Ball Mill. Minerals Engineering, 151, Article ID: 106311. https://doi.org/10.1016/j.mineng.2020.106311

[8] Orozco, L.F., Nguyen, D.H., Delenne, J.Y., Sornay, P. and Radjai, F. (2020) Discrete-Element Simulations of Comminution in Rotating Drums: Effects of Grinding Media. Powder Technology, 362, 157-167.

https://doi.org/10.1016/j.powtec.2019.12.014

[9] Jiang, S., Ye, Y., Tan, Y., Liu, S., Liu, J., Zhang, H. and Yang, D. (2018) Discrete Element Simulation of Particle Motion in Ball Mills Based on Similarity. Powder Technology, 335, 91-102. https://doi.org/10.1016/j.powtec.2018.05.012

[10] Powell, M.S., Weerasekara, N.S., Cole, S., LaRoche, R.D. and Favier, J. (2011) DEM Modelling of Liner Evolution and Its Influence on Grinding Rate in Ball Mills. Minerals Engineering, 24, 341-351. https://doi.org/10.1016/j.mineng.2010.12.012

[11] Stoimenov, N. and Ruzic, J. (2019) Analysis of the Particle Motion during Mechanical Alloying Using EDEM Software. IFAC-PapersOnLine, 52, 462-466.

https://doi.org/10.1016/j.ifacol.2019.12.583

[12] Ueda, S., Natsui, S., Fan, Z., Nogami, H., Soda, R., Kano, J., Noue, R. and Ariyama, T. (2010) Influences of Physical Properties of Particle in Discrete Element Method on Descending Phenomena and Stress Distribution in Blast Furnace. ISIJ International, 50, 981-986. https://doi.org/10.2355/isijinternational.50.981

[13] Cleary, P.W., Morrison, R. and Morrell, S. (2003) Comparison of DEM and Experiment for a Scale Model SAG Mill. International Journal of Mineral Processing, 68, 129-165. https://doi.org/10.1016/S0301-7516(02)00065-0

[14] Cleary, P.W. and Hoyer, D. (2000) Centrifugal Mill Charge Motion: Comparison of DEM Predictions with Experiment. International Journal of Mineral Processing, 59, 131-148. https://doi.org/10.1016/S0301-7516(99)00063-0

[15] Govender, I., Cleary, P.W. and Mainza, A.N. (2013) Comparisons of PEPT Derived Charge Features in Wet Milling Environments with a Friction-Adjusted DEM Model. Chemical Engineering Science, 97, 162-175. https://doi.org/10.1016/j.ces.2013.04.023

[16] Cleary, P.W. (2015) A Multiscale Method for Including Fine Particle Effects in DEM Models of Grinding Mills. Minerals Engineering, 84, 88-99.

https://doi.org/10.1016/j.mineng.2015.10.008

[17] Mhadhbi, M. (2021) Modelling of the High-Energy Ball Milling Process. Advances in Materials Physics and Chemistry, 11, 31-44. https://doi.org/10.4236/ampc.2021.111004

[18] Williams, J.R. and Pentland, A. (1992) Superquadrics and Modal Dynamics for Discrete Elements in Interactive Design. International Journal of Computer-Aided Engineering-Engineering Computations, 9, 115-127. https://doi.org/10.1108/eb023852

[19] Cleary, P.W. (2004) Large Scale Industrial DEM Modelling. Engineering Computations, 21, 169-204. https://doi.org/10.1108/02644400410519730

[20] Cleary, P.W. (2001) Charge Behaviour and Power Consumption in Ball Mills: Sensitivity to Mill Operating Conditions, Liner Geometry and Charge Composition. In- 
ternational Journal of Mineral Processing, 63, 79-114.

https://doi.org/10.1016/S0301-7516(01)00037-0

[21] Hirosawa, F., Iwasaki, T. and Iwata, M. (2021) Particle Impact Energy Variation with the Size and Number of Particles in a Planetary Ball Mill. MATEC Web of Conferences, 333, 1-5. https://doi.org/10.1051/matecconf/202133302016

[22] Daraio, D., Villoria, J., Ingram, A., Alexiadis, A., Stitt, E.H., Munnoch, A.L. and Marigo, M. (2020) Using Discrete Element Method (DEM) Simulations to Reveal the Differences in the $\gamma-\mathrm{Al}_{2} \mathrm{O}_{3}$ to $\alpha-\mathrm{Al}_{2} \mathrm{O}_{3}$ Mechanically Induced Phase Transformation between a Planetary Ball Mill and an Attritor Mill. Minerals Engineering, 155, Article ID: 106374. https://doi.org/10.1016/j.mineng.2020.106374 